\title{
TRATAMENTO CIRÚRGICO DAS LESÕES TRAUMÁTICAS DO SEGMENTO MÉDIO-INFERIOR DA COLUNA CERVICAL
}

\author{
MARCO ANTONIO HERCULANO*, OSWALDO INÁCIO DE TELLA JÚNIOR** \\ ANTONIO DE PÁDUA FURQUIM BONATELLI***
}

\begin{abstract}
RESUMO - O nível C2 é o mais frequente em lesões traumáticas da coluna cervical, sendo seguido por C5 e C6. Elas ocorrem, mais habitualmente, na terceira década de vida, com incidência decrescente na idade avançada. Como parte destas lesões está localizada nos elementos da coluna anterior, segundo a conceituação de Denis e, levado-se em consideração os estudos biomecânicos do segmento médio-inferior da coluna cervical, bem como os mecanismos que causam estas lesões, elaboramos este estudo, com o intuito de apresentar as técnicas de fusão cervical anterior, com enxerto ósseo e placas de Caspar, Morscher (CSLP) e ORIONTM. Mostramos e discutimos as vantagens e desvantagens de cada tipo de fixação anterior, com base nos dados de literatura, evidenciando a melhoria no material de implante e das técnicas de fixação.
\end{abstract}

PALAVRAS-CHAVE: coluna, lesões traumáticas, cirurgia, região cervical, traumatismo raquimedular cervical.

\section{Surgical treatment of the traumatic lesions of medium-inferior segment of cervical column}

ABSTRACT - The most frequently injury level of cervical spine is C2, followed by C5 and C6. Injuries were most commonly sustained in the third decade of life, with a decreasing incidence in the advanced age. As part of these lesions is located in the elements of the anterior column, according to Denis's definition and, taking in consideration the biomechanics studies of the segment medium-inferior of the cervical spine, as well as the mechanisms that cause their lesions, this study was elaborated, with the intention of presenting the techniques of anterior cervical fusion, with bony graft and plates of Caspar, Morscher (CSLP) and ORIONTM. The advantages and disadvantages of each type of anterior fixation are presented accordind to the literature review. The improvement in the material relate to plates and screws fixation techniques are illustrated.

KEY WORDS: spine, traumatic injury, surgery, cervical spine, cervical spine injury.

As lesões da coluna vertebral têm sido descritas desde os tempos mais remotos. A primeira referência a fraturas da coluna vertebral esta escrita no papiro de Edwin Smith ${ }^{1}$, em 2500 a.C. Sonntag $^{1}$, relata que Celsus (30 a.C.) notava que a morte se seguia rapidamente às lesões vertebrais envolvendo a região cervical e afirma que Galeno (130-201) provocava, experimentalmente, a interrupção da medula levando a paralisia e perda da sensibilidade abaixo do nível da lesão. Relata ainda descrições de tratamentos cirúrgicos preconizados por Ambroise Paré (1510-1590) e Fabricius Hildanus (1646). As modernas técnicas de tratamento das lesões coluna da cervical tiveram seu início em 1891 quando $\mathrm{Hadra}^{2}$ descreve uma técnica de fusão, através de amarrilho dos processos espinhosos, para o tratamento do mal de Pott.

Resumo de tese apresentada junto à Universidade Federal de São Paulo (UNIFESP) - Escola Paulista de Medicina (EPM), para a obtenção do título de Mestre em Neurocirurgia: *Mestre em Neurocirurgia pela EPM UNIFESP **Professor Adjunto da Discipina de Neurocirurgia do Departamento de Neurologia da EPM UNIFESP; ***Professor Adjunto da Discipina de Neurocirurgia do Departamento de Neurologia da EPM UNIFESP. 
A fusão vertebral, senso estrito, se refere aos procedimentos efetuados nos elementos ósseos da coluna, fornecendo continuidade a um número específico destes segmentos. Por esta definição, a história da fusão vertebral se iniciou na cidade de Nova Iorque, em 1911, quando Albee ${ }^{3}$ apresenta seus casos, usando enxertos da cortical de tíbia. Em 1976, White et al. ${ }^{4}$ demonstraram, experimentalmente, que, em lesão posterior, a fusão deve ser posterior e vice-versa, fato este confirmado, clinicamente, em 1977, por Stauffer \& Kelly ${ }^{5}$.

A porcentagem de instabilidade, no tratamento conservador de luxações da coluna cervical com imobilização após redução incruenta, varia entre $5,4 \%^{6}$, e 70,0\% , em pacientes portadores de luxações ou fraturas-luxações. Aebi et al. ${ }^{8}$, preconizam o procedimento cirúrgico na coluna cervical para evitar maior dano à medula espinhal ou raízes nervosas, e restauração da estabilidade. A via de acesso a ser utilizada pode ser anterior, posterior ou a combinação de ambas, quando uma, isoladamente, não fornecer estabilidade e descompressão suficiente. Ao contrário das lesões $\mathrm{C}_{1}-\mathrm{C}_{2}$, nas quais o tratamento é geralmente padronizado, Borre et al. ${ }^{9}$, relatam que as lesões cervicais do segmento médio-inferior $\left(\mathrm{C}_{3}\right.$ $\mathrm{C}_{7}$ ) mostram grande diversidade, fazendo com que a escolha da melhor técnica cirúrgica seja muitas vezes difícil. Kalfas ${ }^{10}$, afirma que várias séries têm demonstrado o sucesso da fusão anterior, variando de 74,0 a $98,0 \%$. A falha na fusão, deslocamento do enxerto e deformidade pós-operatória da coluna cervical estimularam o desenvolvimento das placas de fixação para coluna vertebral. Herrmann ${ }^{11}$, em 1975, descreve pela primeira vez o uso de placa de metal para fixação cervical anterior.

Por tudo o que se pode depreender do exposto, as lesões traumáticas da coluna vertebral, em seu segmento médio-inferior, apresentam-se como um desafio aos cirurgiões que enveredam neste caminho, sendo que dia a dia se multiplicam os estudos de diversas técnicas e materiais cirúrgicos para a definição do melhor tratamento dessa doença.

Este estudo visa apresentar as técnicas de fixação anterior com suas evoluções, relacionadas a uma melhoria no material de implante e na maneira de sua fixação. Sabe-se, no entanto, que existe hoje um número muito grande de novos implantes e substitutos de enxerto homólogo que necessitam de estudos biomecânicos mais elaborados para uma análise do comportamento in vitro e in vivo, bem como a resistência biomecânica, imediata, dos sistemas.

Em 1984, Denis ${ }^{12,13}$ apresenta novo conceito dividindo a coluna vertebral em "três colunas": 1) coluna posterior: formada pelo arco ósseo posterior, ligamentos supra-espinhoso, interespinhoso, cápsula articular e ligamento amarelo; 2) coluna média: formada pelo ligamento longitudinal posterior, ânulo fibroso posterior e a parte posterior do corpo vertebral; 3) coluna anterior: formada pelo ligamento longitudinal anterior, o ânulo fibroso anterior e parte anterior do corpo vertebral. Ele classifica os principais tipos de fraturas da coluna em categorias, definidas em termos do grau de envolvimento de cada uma das colunas: 1) fratura por compressão: falha sob compressão da coluna anterior. A coluna média intacta, funcionando como dobradiça, tenciona a coluna posterior na qual ocorre falha parcial. Descreve a importância da coluna média intacta para este tipo, evitando a retropulsão de fragmentos, luxação posterior e compressão dos elementos neurais; 2) fraturas tipo explosão: falha por carga axial das colunas anterior e média. Devido a diferentes tipos de envolvimento da placa terminal descreve os subtipos: fratura envolvendo ambas as placas terminais; fratura da placa superior; fratura da placa terminal inferior; fratura do tipo explosão com rotação; 3 ) fraturas do tipo "cinto de segurança": falha das colunas média e posterior pela tensão gerada através do movimento de flexão, tendo como eixo do movimento a coluna anterior, a qual pode falhar parcialmente; 4) fraturas luxações: falha das três colunas sob compressão, tensão, rotação ou cisalhamento. São as mais instáveis. Refere-se a três subtipos de fratura-luxação: flexão-rotação, cisalhamento e flexão-distração.

\section{MÉTODO}

\section{Técnica}

Em 1958, Smith e Robinson ${ }^{14}$ descrevem o procedimento cirúrgico e o resultado da remoção do disco intervertebral com fusão da coluna cervical pela via anterior em 14 pacientes que apresentavam alterações osteoartríticas, causando irritação da raíz nervosa, concluindo que este método aliviou completamente o desconforto de nove dos 14 pacientes e ocasionou melhora clínica em quatro casos, sem ter acarretado complicações graves. 
Tabela 1. Relação dos doentes, número de registro, iniciais, gênero, idade, nível da lesão.

\begin{tabular}{ccccc}
\hline Registro & Iniciais & Gênero & Idade (anos) & Nível da lesão \\
\hline CMHP & LB & Masculino & 19 & C6 \\
$50.720 / 01 \mathrm{HPS}$ & CI & Masculino & 13 & C6 \\
$28.868 / 01 \mathrm{HPS}$ & ATS & Masculino & 19 & $\mathrm{C} 5$ \\
$47.947 \mathrm{CMHP}$ & MSS & Masculino & 26 & $\mathrm{C} 6$ \\
$47.611 \mathrm{CMHP}$ & MMM & Feminino & 28 & $\mathrm{C} 7$ \\
CETOM & JAS & Masculino & 22 & C6 \\
52.451 CMHP & AEL & Masculino & 35 & C5 \\
HPEV & NVS & Masculino & 29 & C5 \\
52.728 CMHP & RAM & Masculino & 17 & C6 \\
CMHP & ARG & Feminino & 17 & C4 \\
\hline
\end{tabular}

CMHP, Centro Médico Hospitalar das Pitangueiras; CETOM, Hospital Evangélico; HPS, Hospital Paulo Sacramento; HPEV, Hospital Prof. Edmundo Vasconcelos. $\mathrm{n}=10$

Bailey e Badgley ${ }^{15}$, salientam que a fusão anterior pode ser uma alternativa atraente em relação à fusão posterior. As indicações para sua utilização incluem a lesão destrutiva do corpo vertebral propiciando retirada de material para biópsia, remoção da lesão e descompressão da coluna restaurando sua estabilidade. Afirmam que ambas as técnicas de fusão, anterior e posterior, são de extremo valor, sendo a seleção de cada procedimento baseada na natureza individual de cada lesão. Hirsch ${ }^{16}$, descreve técnica de abordagem cirúrgica anterolateral através de incisão ao longo da borda do músculo esternocleidomastóideo, passando a frente do músculo esternoclavicular. A artéria carótida é afastada lateralmente e a glândula tireóide, a traquéia e o esôfago medialmente, obtendo-se a exposição da região ventrolateral do disco intervertebral. Relata não fazer uso de enxerto ósseo.

Em 1958, Cloward ${ }^{17,18}$ publica artigo sobre seu método de tratamento por via anterior. O procedimento cirúrgico inclui redução anterior da luxação com descompressão da medula e raízes nervosas bem como a fusão dos corpos vertebrais. Afirma que, após ocorrer a fusão intersomática, desenvolveu-se angulação anterior da coluna cervical em 50,0\% dos pacientes, provavelmente devido à força compressiva aplicada na parte anterior do corpo vertebral que lesa o enxerto ósseo, resultando em enfraquecimento da capacidade de suporte de peso no plano vertical. Observa que a fusão sólida se fez presente em todos os casos, apesar da alta incidência de pacientes que apresentam alteração no alinhamento normal da coluna cervical.

Robinson, em 1964, publica estudo relativo às fusões da coluna cervical por via anterior e posterior. Relata as seguintes vantagens da fusão anterior sobre a fusão posterior: 1) maior facilidade técnica em sua realização; 2) menor morbidade cirúrgica, já que a via de acesso é realizada entre músculos cervicais; 3) capacidade de restabelecer alinhamento anatômico da coluna cervical, quando isto não é alcançado por via posterior e 4) obtenção de taxa razoavelmente alta de artrodese $(88,0 \%)^{19}$.

Tabela 2. Distribuição de frequência segundo a faixa de idade.

\begin{tabular}{ccc}
\hline Idade (anos) & Frequência & $\%$ \\
\hline $10-20$ & 5 & 50,0 \\
$20-30$ & 4 & 40,0 \\
$30-40$ & 1 & 10,0 \\
\hline
\end{tabular}

Idade mínima: 13; idade máxima: 35; idade média: 22,5 ( $\pm 6,8)$. 


\section{Casuística}

Nosso material foi composto de 10 doentes portadores de traumatismo da coluna cervical, com lesões nos elementos da coluna anterior, média, posterior e combinadas. A idade média dos doentes foi 22,5 anos, com desvio padrão de 6,6. O nível da lesão variou entre C5 a C7, com maior incidência em C6 com 10 casos (50,0\%), como mostra a Tabela 1 . Houve predomínio no gênero masculino, 8 casos $(80,0 \%)$, em relação ao gênero feminino, 2 casos $(20,0 \%)$, como se observa na Tabela 2.

Seis doentes tiveram suas lesões corrigidas através da fixação anterior com placa e parafusos e dois doentes foram submetidos à fixação externa com colar cervical. Dois doentes que apresentavam lesões combinadas dos elementos da coluna anterior e posterior foram submetidos à fixação anterior com enxerto ósseo, placa e parafusos, associada à fixação posterior pela técnica de amarrilho interespinhoso. A seguir apresentamos um de nossos casos de especial interesse dada sua complexidade.

AEL - masculino, 35 anos de idade, internado em 4 de janeiro de 1999 (Tabela 1). Durante estadia no litoral mergulhou batendo com a cabeça na areia. Procurou atendimento médico no local sendo dispensado com o uso de antiinflamatórios. Retornou a sua origem, após vinte e quatro horas, com dor cervical intensa. Procurou novo serviço onde foi diagnosticada luxação cervical C5/C6, sendo submetido à fixação anterior com corpectomia de C5 e colocação de "cage" de titânio com placa e parafusos do sistema CLSP. Após alta hospitalar, no décimo dia de pós-operatório, procurou nosso serviço com intensa dor em membros superiores e a radiografia de coluna cervical mostrava perda do alinhamento e do sistema de fixação. Foi internado e submetido, no mesmo ato cirúrgico, a tração esquelética cervical com retirada do sistema de fixação e "cage" e colocação de enxerto homólogo, de crista ilíaca anterior, e fixação com placa e parafuso do sistema SICEL®, seguido de fixação posterior por amarrilho interespinhoso entre C4-C5 e C5-C6, com fios de titânio (Figs 1, 2, 3).

\section{RESULTADOS E DISCUSSÃO}

A fixação anterior com placa avançou quando Caspar $^{20}$, introduziu um novo sistema de placa para fixação anterior da coluna cervical. Este sistema consiste de conjunto estandardizado e universal de instrumentos, retratores, distratores e placas de osteosíntese com parafusos de fixação de tamanho variado. O sistema de CASPAR quando associado a enxerto ósseo promove a estabilização imediata

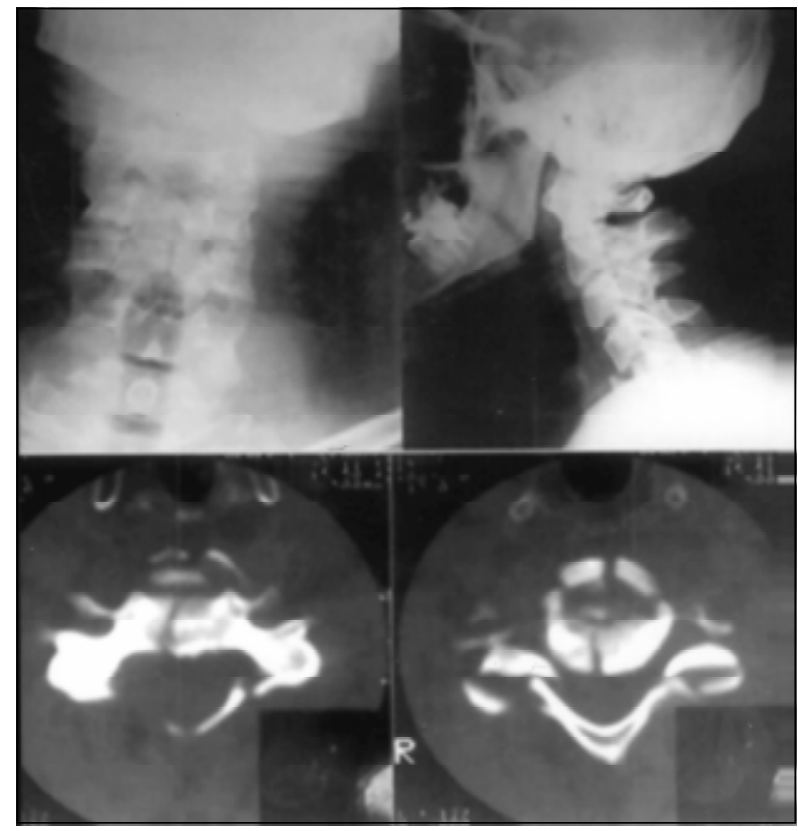

Fig 1. Radiografia em anteroposterior e perfil mostrando a subluxação de C6 com fratura do corpo vertebral confirmada pela tomografia. 


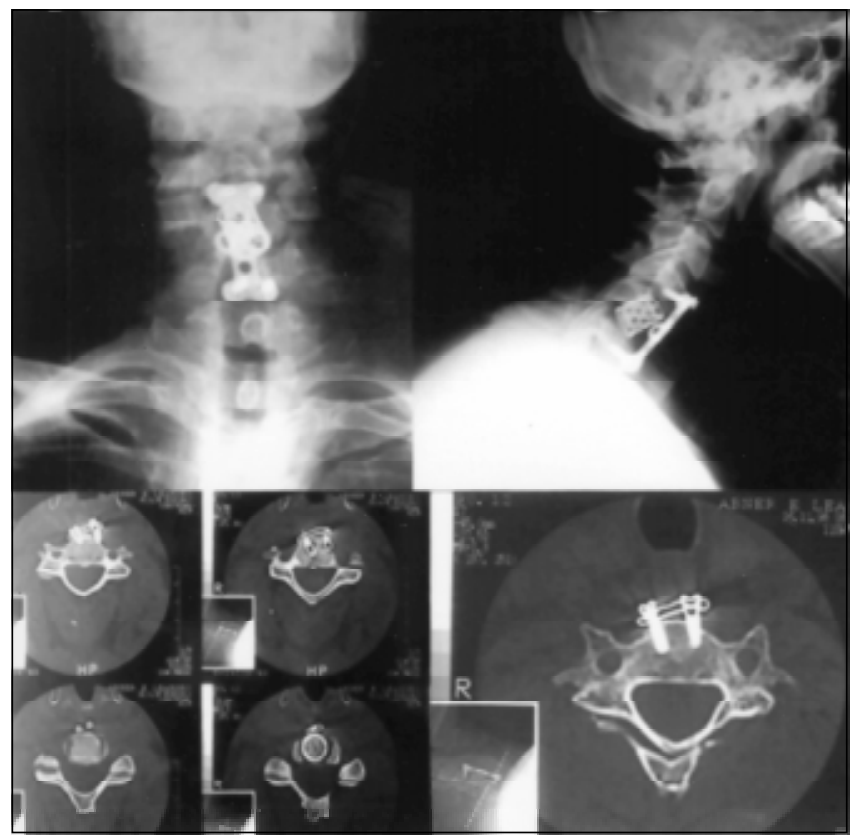

Fig 2. Radiografias em anteroposterior e perfil mostrando a subluxação com perda do alinhamento cirúrgico e da fixação da placa, confirmadas pelos cortes da tomografia.

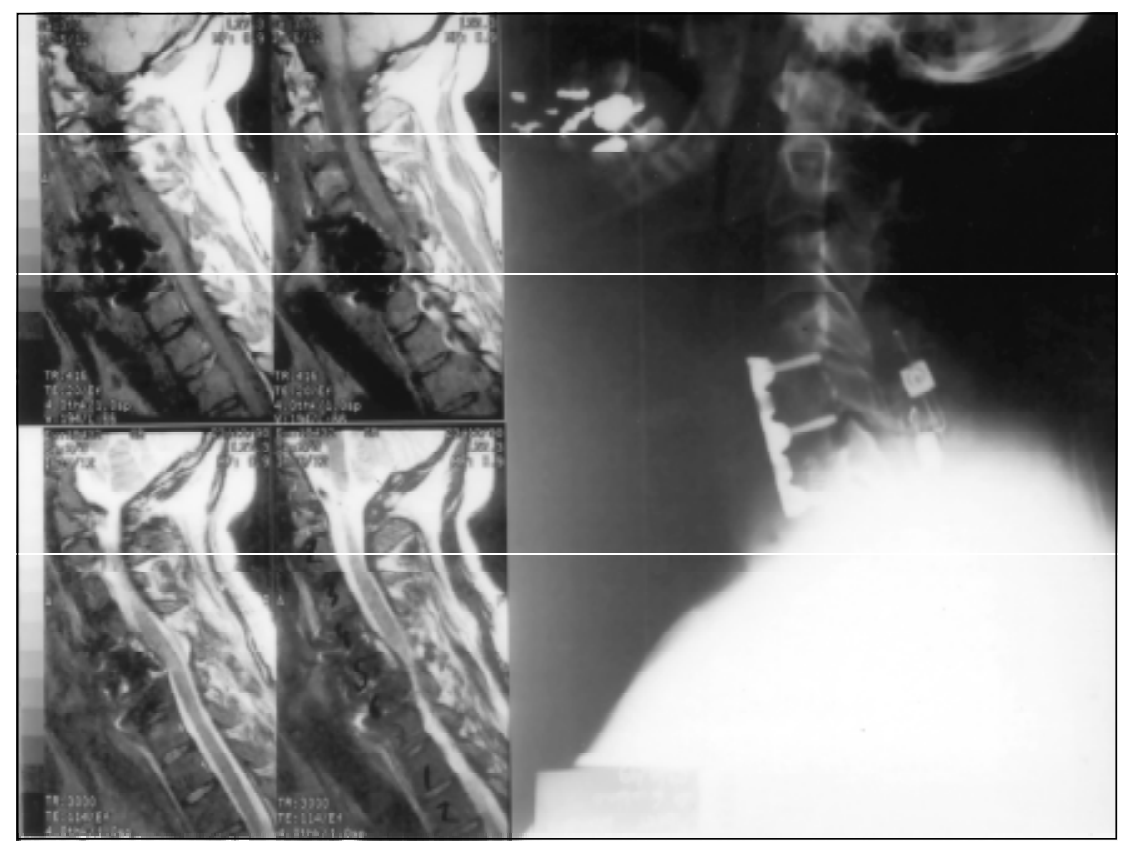

Fig 3. Ressonância magnética mostrando a compressão medular pela “cage” e radiografia em perfil mostrando a fixação anterior e posterior combinada. 
da coluna sem a necessidade de imobilização por halo no pós-operatório segundo Kalfas ${ }^{21}$. O sistema apresenta todos os critérios para a ótima cura da fratura, ou incorporação do enxerto; especificamente, realinhamento anatômico, contato osso a osso, absoluta imobilização e compressão dos fragmentos ósseos ou do enxerto. A desvantagem é que o sistema requer que o parafuso usado na fixação atravesse a córtex anterior e a posterior do corpo vertebral. Isto acrescenta um risco de lesão neurológica, caso a broca ou o parafuso passe além da córtex posterior do corpo vertebral, em direção ao canal vertebral.

Moscher et al. ${ }^{22}$, introduziram um sistema de placa anterior com vantagem mecânica na fixação interna, reduzindo o risco de lesão neurológica, associada à fixação do parafuso na córtex anterior do corpo vertebral. O sistema de parafuso travado na placa cervical (CSLP) usa um parafuso de ancoragem com um canal, tornando sua cabeça expansível. Depois de inserido este parafuso, um segundo parafuso travante é colocado na cabeça daquele. Este parafuso distende a cabeça do parafuso de ancoragem comprimindo-o contra a parede do orifício da placa. Este tipo de fixação elimina a necessidade de se passar o parafuso através da córtex posterior do corpo vertebral.

Lowery $^{23}$, descreveu um novo sistema de fixação, variante do sistema de Moscher, o Orion ${ }^{\mathrm{TM}}$, sistema de placa cervical anterior. Neste sistema o parafuso de ancoragem se fixa em uma depressão na superfície superior da placa e um parafuso travante é aplicado sobre o mesmo.

As técnicas de estabilização da coluna cervical apresentaram avanço significativo durante a última década. Melhor compreensão da biomecânica da coluna vertebral permitiu o desenvolvimento de inúmeras técnicas de fixação interna para complementar ou substituir as técnicas existentes. Apesar de terem sido desenvolvidas para o tratamento da instabilidade das lesões traumáticas da coluna cervical, atualmente estas técnicas são utilizadas para a estabilização das doenças degenerativas, tumorais e instabilidade cervical. O objetivo primário do tratamento da instabilidade cervical é minimizar tanto a lesão neurológica quanto a dor decorrente da deformidade da coluna, promovendo a recuperação da medula e raízes nervosas ${ }^{10,15,18,24}$, levando à estabilidade do complexo ósteo-músculo-ligamentar que irá prevenir outras lesões dos elementos neurais.

Uma grande variedade de técnicas de fixação e instrumentação é usada no tratamento das lesões traumáticas, doenças degenerativas, tumor e infeções da coluna cervical. A técnica a ser utilizada depende do paciente, do tipo de lesão, do grau de instabilidade e experiência do cirurgião, segundo Slone et al. ${ }^{25}$. O tratamento das fraturas-luxações do segmento médio-inferior, através da fixação interna e fusão precoce da coluna cervical, promoveu menor deformidade residual, baixa taxa de recorrência, menor tempo de permanência hospitalar, retorno mais precoce às atividades de trabalho, além de permitir dispensar o uso de órteses, que muitas vezes são desconfortáveis para o paciente, promovendo estabilidade imediata como afirmam Forsyth et al. ${ }^{26}$, e Kalfas ${ }^{10}$, até que ocorra a fusão definitiva. A decisão de estabilizar ou não a coluna cervical e o método a ser utilizado deve ser baseado em cada caso individualmente. Em lesões instáveis, o local da instabilidade influencia a escolha da via de acesso. A instabilidade pode ser anterior ou posterior, dependendo do mecanismo do trauma. A coluna cervical tem a característica inerente de ter a maior mobilidade e os segmentos menos estáveis de toda a coluna vertebral. A estabilização precoce diminui a morbidade dessas lesões, segundo Stauffer e Rhoades ${ }^{27}$.

As técnicas usadas no tratamento cirúrgico das lesões da coluna cervical podem ser divididas em anteriores, posteriores e combinadas ou circulares, como afirmam Van Peteghem e Schweigel ${ }^{28}$, e Slone et al. ${ }^{25}$. A instabilidade posterior deve ser tratada por fusão posterior e instabilidade anterior por fusão anterior, de acordo com Stauffer e Kelly5. Quando ocorre lesão discoligamentar completa, a fixação circular ou combinada é preconizada para se obter uma estabilização adequada, segundo Ulrich et al. ${ }^{29}$.

Concordamos com Cabanela e Ebersold ${ }^{30}$, que afirmam ser a estabilização com placa após corpectomia anterior um bom método para prevenir o deslocamento do enxerto em fraturas instáveis gota de lágrima da coluna cervical. 
Nosso estudo tem por finalidade apresentar as técnicas de fixação anterior da coluna cervical, muito empregadas por neurocirurgiões, analisando as diversas placas com seus sistemas de fixação (Caspar, Morscher e Lowery). Na análise dos resultados do tratamento cirúrgico por via anterior, com uso de placas de Caspar e sistema CSLP, em corpectomias isoladas ou em vários níveis para tratamento de espondilose, trauma, tumor do corpo vertebral e cifoses pós-traumáticas ou póslaminectomias, nota-se que a diferença primária reside no tamanho do parafuso do corpo vertebral. Os parafusos usados no sistema de Caspar variam de comprimento, mas necessitam ser fixadas nas paredes corticais anterior e posterior do corpo vertebral. Caso não sejam apropriadamente posicionados, intra-operatoriamente, a ponta dos parafusos pode penetrar no canal medular como afirma Kalfas ${ }^{10}$. Os parafusos usados no sistema CSLP necessitam ser fixados somente na parede cortical anterior do corpo vertebral e têm usualmente de $14 \mathrm{~mm}$ de comprimento, existindo parafusos menores para uso pediátrico.

O estudo biomecânico, in vitro, comparativo entre as placas de Caspar e o sistema CSLP de Morscher para fixação anterior da coluna cervical, mostra que o sistema CSLP é preferível às placas trapezoidal de Caspar para restabelecer a estabilidade da coluna cervical em flexão, devido ao menor risco de soltar o parafuso com deslocamento da placa como nos afirmam Smith et al. ${ }^{31}$.

\section{Conclusões}

Em vista dos elementos apresentados, podemos inferir que:

Nas lesões da coluna anterior, a fixação anterior é o tratamento cirúrgico de escolha para as lesões traumáticas do segmento médio-inferior da coluna cervical.

O sistema de parafuso travado na placa cervical (CSLP) oferece menor risco de lesão neurológica e de perda da fixação do parafuso, se comparado ao sistema de fixação com placa e parafusos bicorticais.

A fixação anterior associada ao enxerto ósseo promove estabilidade imediata, tornando desnecessário o uso de imobilização externa no pós-operatório.

\section{REFERÊNCIAS}

1. Sonntag VKH. History of spinal disorders. In Menezes AH, Sonntag VKH. Principles of spinal surgery. New York: McGrawHill, 1996:1-23.

2. Hadra BE. Wiring of the vertebrae as a means of immobilization in fracture and Potts' disease. Medical Times and Register 1891;22:423-429.

3. Albee FH. Transplantation of portions of the tibia into the spine for Pott's desease. JAMA 1911;57:885.

4. White AA III, Southwick WO, Panjabi MM. Clinical instability in the lower cervical spine: a review of past and concepts. Spine 1976;1:15-27.

5. Stauffer ES, Kelly EG. Fracture-discolations of the cervical spine: instability and recurrent deformity following treatment by anterior interbody fusion. J Bone Joint Surg 1977;59:45-48.

6. Cheshire DJE. The stability of the cervical spine following the conservative treatment of fractures and fracture-discolations. Paraplegia 1969;7:193-203.

7. Kalff R, Kocks W, Roossen K, Schmit-Neuerburg KP, Birg HW. Die belastungsstabile Spondylodese bei Verletzungen der unteren Halswirbelsäule. Neurochirurgia 1988;31:179-185.

8. Aebi M, Mohler J, Zäch GA, Morscher, E. Indication, surgical technique, and results of 100 surgically-treated fractures and fracture-discolations of the cervical spine. Clin Orthop 1986;23:244-257.

9. Borre G, Bedou G, Pineaudeau M, Omeiri SE, Cristino G. Treatment of severe lesions of the lower cervical spine (C3-C7). Neurochirurgia 1988;31:1-13.

10. Kalfas IH. Cervical spine stabilization: surgical techniques. Neuroimag Clin N Am 1995;5:491-505.

11. Herrmann HD. Metal plates fixation after anterior fusion of unstable facture dislocation of the cervical spine. Acta Neurochir (Wien) 1975;32:101-111.

12. Denis F. Spinal instability as defined by the three column spine concept in acute spinal trauma. Clin Orthop Relat Res 1984;189:65-76.

13. Denis F. The three column spine and its significance in the classificaton of acute thoracolumbar spinal injuries. Spine 1983;8:817-831.

14. Smith GW, Robinson RA. The treatment of certain cervical-spine disorders by anterior removal of the intervertebral disc and interbody fusion. J Bone Joint Surg 1958;40:607-624.

15. Bailey RW, Badgley CE. Stabilization of the cervical spine by anterior fusion. J Bone Joint Surg 1960;42:565-594.

16. Hirsch C. Cervical disk rupture: diagnosis and therapy. Acta Orthop Scand 1960;30:172-186. 
17. Cloward RB: The anterior approach for removal of ruptured cervical disks. J Neurosurg 1958;15:602-614.

18. Cloward RB. Treatment of acute fractures and fracture-discolations of the cervical spine by vertebral-body fusion. $\mathrm{J}$ Neurosurg 1961;18:201-209.

19. Robinson RA. Anterior and posterior cervical spine fusions. Clin Orthop 1964;35:34-62.

20. Caspar W. Anterior cervical fusion and interbody stabiization with the trapezial osteosyntethic plate technique. Aesculap Scientific Information Leaflet S-039. Burlingame, CA, Aesculap Instruments Corp, 1986.

21. Caspar W, Barbier DD, Klara PM. Anterior cervical fusion and CASPAR plate stabilization for cervical trauma. Neurosurgery 1989;25:491-502.

22. Morscher E, Sutter F, Jenny H, Olerud S. Die vordere Verplattung der Halswirbelsäule mit dem Hohlschrauben-Plattensystem aus Titanium. Der Chirurg 1986;57:702-707.

23. Lovery LG. Anterior cervical osteosynthesis: Orion ${ }^{\mathrm{TM}}$ anterior cervical plate system. In Hitchon PW, Traynelis VC, Rengachary S. Techniques in spinal fusion and stabilization. New York: Thieme, 1995:191-197.

24. Holdsworth F. Fractures, dislocations, and fracture-dislocations of the spine. J Bone Joint Surg 1970;52:1534-1551.

25. Slone RM, McEnery KW, Bridewell KH, Montgomery WJ. Fixation techniques and instrumentation used in the cervical spine. Radiol Clin N Am 1995;33:213-230.

26. Forsyth HF, Alexander E, Davis C, Underdal R. The advantages of early spine fusion in the treatment of fracture: discolation of the cervical spine. J Bone Joint Surg 1959;41:17-36.

27. Stauffer ES, Rhoades ME. Surgical stabilization of the cervical spine after trauma. Arch Surg 1976;111:652-657.

28. Van Peteghem PK, Schweigel JF. The fractured cervical spine rendred unstable by anterior cervical fusion. J Trauma 1979;19:110-114.

29. Ulrich C, Worsdörfer O, Claes L, Magerl F. Comparative study of the stability of anterior and posterior cervical spine fixation procedures. Arch Orthop Trauma Surg 1987;106:226-231.

30. Cabanela ME, Ebersold MJ. Anterior plate stabilization for bursiting teardrop fractures of the cervical spine. Spine 1988;13:888-891.

31. Smith AS, Lindsey RW, Doherty BJ, Alexander J, Dickson JH. An in-vitro biomechanical comparison of the Orosco and AO locking plates for anterior cervical spine fixation. J Spinal Disord 1995;8:220-223. 